KFA-IKP(TH)-1997-09

6 May 1997

\title{
Coherent neutrino magnetic conversion in crystals.
}

\author{
V.R.Zoller \\ Institut für Kernphysik, Forschungszentrum Jülich, \\ D-52425 Jülich, Germany 它 \\ Institute for Theoretical and Experimental Physics, \\ ul. B.Chermushkinskaya 25, 117218 Moscow, Russia 月
}

\begin{abstract}
We study coherent enhancement of neutrino electro-magnetic conversion in crystals. Large coherence length which grows with the neutrino energy makes the coherent enhancement particularly effective for very small masses. We derive constraints on the conversion rate which follow from the Fresnel effects in scattering of neutrinos on atomic chains. We comment on possible applications of the crystal converter to searches for non-diagonal neutrino magnetic transitions in the CERN neutrino beam.
\end{abstract}

\footnotetext{
${ }^{1}$ KPH166@AIX.SP.KFA-JUELICH.DE

2 ZOLLER@HERON.ITEP.RU
} 
In [1] the multiple scattering theory of Coulomb coherent excitation of high-energy particles propagating through a crystal was developed. The coherent resonance enhancement of rare processes like the weak radiative transition $p \gamma \rightarrow \Sigma^{+}$[2] has been studied in detail. In the plane wave Born approximation one would expect the transition amplitude on a chain of $N$ identical atoms $\mathcal{T}_{\Sigma^{+} p} \propto N$ for the beam momentum adjusted to satisfy the coherency condition at a given lattice spacing $d$ [3] (for earlier discussions on coherent enhancement of atomic transitions see [4]). The important finding of Ref. [1] is that distortions by the initial/final state interactions of the proton/sigma with a Coulomb field of a crystal suppress the coherent enhancement dramatically: $\mathcal{T}_{\Sigma^{+} p} \propto \log N$ already at $N \gtrsim(Z \alpha)^{-1}$.

Still another interesting process is the neutrino magnetic conversion [5] in a Coulomb field of crystals

$$
\nu_{1} \gamma \rightarrow \nu_{2}
$$

Hereafter, $\nu_{1}$ and $\nu_{2}$ are the neutrino mass eigenstates. In this case the initial/final state interaction effects are negligible for all practical purposes and the law

$$
\mathcal{T}_{21}=t_{21} N
$$

is expected to hold [1] , where $t_{21}$ is the transition amplitude on an isolated atom. Recently there was much discussion on possible laboratory observations of neutrino electro-magnetic conversion, for instance, using the high-quality resonant cavity converters [6, 7, 8]. In these experiments one deals with transition form factor at the photon virtuality as small as $q^{2} \sim\left(10^{-6} e V\right)^{2}$. The bounds on the neutrino magnetic moment which comes from the high-energy $\nu_{\mu} e$ elastic scattering correspond to quite different scale $q^{2} \sim(100 \mathrm{MeV})^{2}$. A possibility of the coherent enhancement of neutrino transitions in crystal converters must not be overlooked since it provides the information on still another region of $q^{2} \sim(1-10 \mathrm{KeV})^{2}$. Exploring this region of $q^{2}$ with crystal converters is topical in view of a possible nontrivial $q^{2}$ dependence, resulting in a strong low-momentum enhancement of a neutrino magnetic form factor. A possible mechanism of such enhancement at very small $q^{2}$ was discussed in [9].

In this communication based on the multiple scattering theory we discuss the salient properties of crystal converters. We derive the upper bound for the crystal thickness $L=N d$ at which the conversion takes place on the crystal as a whole. This bound derives from the Fresnel corrections to the conventional Glauber-Gribov approximation [10, 11]. We conclude that crystal converters in the current CERN neutrino beam can provide useful bounds for the transition magnetic and electric dipole moments of the neutrino.

We start with the brief overview of formalism developed in [1]. The amplitude of coherent transition (11) on a chain built out of $N$ identical atoms is written down as follows

$$
\mathcal{T}_{21}(\mathbf{q})=t_{21}\left(\mathbf{q}_{\perp}\right)\langle N|\exp (i \mathbf{q r})| N\rangle
$$

where $|N\rangle$ is the ground-state wave function of the $\mathrm{N}$-atomic chain. For our purposes it is sufficient to use the uncorrelated wave function of the Gaussian form (see [1] for more detail). Notice that the inter-atomic distances are large compared to the Thomas-Fermi screening radius of the atom, $d \gg a_{T F}$.

From the phenomenological transition matrix element [5]

$$
\mathcal{M}\left(\nu_{1} \gamma \rightarrow \nu_{2}\right)=i \bar{u}_{2}\left(\mu_{21}+\gamma_{5} d_{21}\right) \sigma_{\mu \nu} q^{\nu} \varepsilon^{\mu} u_{1}
$$


one readily finds the helicity-flip amplitude $t_{21}$

$$
t_{21}\left(\mathbf{q}_{\perp}\right)=Z \sqrt{\alpha} \frac{\left[i \mu_{21} \boldsymbol{\sigma}\left[\mathbf{q}_{\perp} \times \mathbf{n}\right]+d_{21} \boldsymbol{\sigma} \mathbf{q}_{\perp}\right]}{q_{\perp}^{2}+\mu^{2}} .
$$

Here $\mu=a_{T F}^{-1}$ and $a_{T F}=\left(m_{e} \alpha Z^{1 / 3}\right)^{-1}$ is the Thomas-Fermi radius of an atom, $\boldsymbol{\sigma}$ is the Pauli spin vector and $\mathbf{n}$ - is a unit vector along the projectile direction. Our choice is $\mathbf{n}=(0,0,1)$. The non-diagonal magnetic and electric dipole moments are denoted by $\mu_{21}$ and $d_{21}$, respectively.

In terms of the matrix element (幽 the cross section of the coherent neutrino conversion in crystal is as follows

$$
\sigma_{21}=\alpha Z^{2} b_{21}^{2} \int d^{2} q_{\perp} \frac{q_{\perp}^{2}}{\left(q_{\perp}^{2}+\mu^{2}\right)^{2}} S_{L}^{2}\left(q_{z}\right) S_{T}^{2}\left(q_{\perp}\right)
$$

In eq.(6) $b_{21}^{2}=\left|\mu_{21}\right|^{2}+\left|d_{21}\right|^{2}$ for Dirac neutrinos and $b_{21}^{2}=4\left[\left(\operatorname{Im} \mu_{21}\right)^{2}+\left(\operatorname{Re} d_{21}\right)^{2}\right]$ for Majorana neutrinos [5].

Denoted by $S_{T}$ is the transverse form factor

$$
S_{T}^{2}\left(q_{\perp}^{2}\right)=\exp \left[-\frac{2}{3}\left\langle\mathbf{u}^{2}\right\rangle q_{\perp}^{2}\right]
$$

where $\left\langle\mathbf{u}^{2}\right\rangle$ is the square of amplitude of the lattice thermal vibrations. It is precisely the factor $S_{T}^{2}$ which cuts off $q_{\perp}^{2}$ at $q_{\perp}^{2} \gtrsim\left\langle\mathbf{u}^{2}\right\rangle^{-1}$.

The longitudinal form factor which involves the structure factor of a crystal is defined as follows

$$
S_{L}\left(q_{z}\right)=\exp \left[-\frac{1}{6} q_{z}^{2}\left\langle\mathbf{u}^{2}\right\rangle\right] \frac{\sin \left(q_{z} N_{z} d / 2\right)}{\sin \left(q_{z} d / 2\right)} .
$$

The transition coherence length is determined by the inverse longitudinal momentum transfer, $L_{c} \sim 1 / q_{z}$. In the very successful eikonal approach to the theory of multiple scattering on extended targets [10, 11] it has been pointed out that $q_{z}=\Delta m_{21}^{2} / 2 p$ [11], where $\Delta m_{21}^{2}=m_{2}^{2}-m_{1}^{2}$. However, the eikonal form of the wave function holds only at distances from the scatterer shorter than the Rayleigh length (see [12 and references therein)

$$
L_{R}=a_{T F}^{2} p
$$

Here $a_{T F}$ is the radius of the scatterer, which in our problem is the Thomas-Fermi radius of an atom: $a_{T F}=\left(m_{e} \alpha Z^{1 / 3}\right)^{-1}$. If the coherence length is larger than the Rayleigh length, the Fresnel corrections to geometrical optics must be included and in the calculation of the converted wave the eikonal Green's function

$$
G_{e i k}(\mathbf{b} ; z)=i \delta(\mathbf{b}) \theta(z) \exp (i p z)
$$

must be substituted by the Fresnel-Green function [12]

$$
G_{F}(\mathbf{b} ; z)=\frac{p \theta(z)}{2 \pi z} \exp \left(\frac{i p \mathbf{b}^{2}}{2 z}\right) \exp (i p z) .
$$

The Fresnel effects are especially important in the high-energy magnetic scattering since the amplitude $t_{21}\left(\mathbf{q}_{\perp}\right)$ vanishes at $q_{\perp} \rightarrow 0$ and the forward scattering dominance [10, 11] 
does not hold. Let $q_{\perp}$ be the transverse momentum of the converted neutrino. Then the converted wave calculated with the Fresnel-Green function acquires the additional Fresnel phase factor $\exp \left[i z q_{\perp}^{2} / 2 p\right]$. Hence

$$
q_{z}=\frac{\Delta m_{21}^{2}+q_{\perp}^{2}}{2 p}
$$

The small-angle scattering regime is assumed to hold, $\theta^{2} \simeq q_{\perp}^{2} / p^{2} \ll 1$.

The coherent conversion rate

$$
R=\frac{\sigma_{21}}{d^{2}}
$$

exhibits the resonance enhancement $R \propto N^{2}$ at the projectile momentum

$$
p \simeq p_{n}=\frac{\left(\Delta m_{21}^{2}+q_{\perp}^{2}\right) d}{4 \pi n}, n= \pm 1, \pm 2, \ldots
$$

which satisfy the equation

$$
q_{z} d=2 \pi n .
$$

As a function of beam momentum $R$ looks like a series of narrow resonances with the width

$$
\Gamma_{n}=\frac{\sqrt{3}}{2} \frac{\left(\Delta m_{21}^{2}+q_{\perp}^{2}\right) d}{\pi^{2} n^{2} N}, \quad n \neq 0 .
$$

The smallness of the ratio $\Gamma_{n} / p_{n} \sim N^{-1}$ leaves little hopes for the observation of these resonances in a wide band neutrino beam. For the practical purposes, one must focus on the coherent enhancement at $n=0$ in eq.([13). In this case, "the resonance width" rises with the neutrino momentum. Indeed, for high energy neutrino $q_{z} d$ is small and the longitudinal form factor in eq.(6)

$$
S_{L}^{2} \simeq N^{2}\left[1-\frac{N^{2}}{N_{c}^{2}}+\ldots\right] .
$$

defines the coherence length $L_{c}=N_{c} d$, where

$$
N_{c}=\frac{4 \sqrt{3} p}{\left(\Delta m_{21}^{2}+q_{\perp}^{2}\right) d} .
$$

One finds the coherent enhancement $R \propto N^{2}$ if $N \ll N_{c}$.

In contrast to the eikonal approximation, where the coherence length is $L_{c}^{e i k} \sim p / \Delta m_{21}^{2}$, the coherence length defined by eq.(16) depends strongly on the transverse momentum $q_{\perp}$. The characteristic $q_{\perp}$ vary in the range $2 \pi / d \lesssim q_{\perp} \lesssim a_{T F}^{-1},\left\langle\mathbf{u}^{2}\right\rangle^{-1 / 2}$. The thermal vibration amplitude estimated from the Debye approximation corresponds to $\left\langle\mathbf{u}^{2}\right\rangle^{-1 / 2} \sim 20-40 \mathrm{KeV}$ for most commonly studied crystals at room temperature [13]. The inverse Thomas-Fermi radius depends on $Z$ and is such that $a_{T F}^{-1} \sim 10-20 \mathrm{KeV}$. Typically, $2 \pi / d \sim 1 \mathrm{KeV}$. These values of $q_{\perp}^{2}$ are much larger than the existing estimates of $\Delta m_{21}^{2}$ based on the observed deficit of solar electron neutrinos, the deficit of atmospheric muon neutrinos relative to electron neutrinos and a relative weight of hot component in the dark matter of the Universe. They vary from $\Delta m_{21}^{2} \sim 10^{-6} \mathrm{eV}^{2}$ to $\Delta m_{21}^{2} \sim 1 \mathrm{eV}^{2}$ ( see [14 for more discussion) and can safely be neglected on the background of lattice vibrations.

As a function of crystal thickness $R$ exhibits fast growth with $L$ which is followed by the saturation regime where $R \simeq 16 \alpha Z^{2} b_{21}^{2} p^{2} / \mu^{4} d^{4}$. Since we are interested in $L$ which are not 
much larger than $L_{c}$, the contribution from the incoherent transitions which is $\propto N$ as well as corrections $\propto \log N$ at $N \gg N_{c}$ are neglected.

The above peculiarities of $R$ are illustrated by Fig. 1 in terms of the non-diagonal transition magnetic moment. We consider the $W$-crystal converter. The attainable sensitivity of the CERN neutrino detectors on the measurement of the neutrino conversion rate in the "disappearance type" experiment is assumed to be $\simeq 10^{-4}$ [6]. Somewhat deliberately we put $\left|\mu_{21}\right|=\left|d_{21}\right|$. So far as $L \ll L_{c}$ the conversion rate follows the geometrical optics. The upper bound on the transition magnetic moment also follows the bound given by the geometrical optics. Fresnel effects enter the game at $L \sim L_{c}$. The variation of the slope in the transitional region is due to the smallness of $\left\langle\mathbf{u}^{2}\right\rangle$ compared to $a_{T F}^{2}$ which is specific for the $W$-crystal [13]. At still larger $L$ the ratio $\mu_{21} / \mu_{B}$ is saturated at the value

$$
\frac{\mu_{21}}{\mu_{B}} \simeq \frac{1}{Z} \frac{m_{e}}{2 p} \frac{d^{2}}{a_{T F}^{2}} \sqrt{\frac{R}{2 \alpha}} .
$$

Here $\mu_{B}$ is the Bohr magneton. The above estimate, eq. (17), does not involve $\left\langle\mathbf{u}^{2}\right\rangle$ since $\left\langle\mathbf{u}^{2}\right\rangle \ll a_{T F}^{2}$. If, on the contrary, $\left\langle\mathbf{u}^{2}\right\rangle \gtrsim a_{T F}^{2}$ the value of $\mu_{21} / \mu_{B}$ in a saturation regime is determined by the effect of thermal vibrations.

In the above analysis the neutrino has been assumed to propagate along the crystallographic axis. In optimization of the conversion rate one must be aware of the natural angular divergence of the neutrino beam. If the projectile momentum $\mathbf{p}$ has non-zero transverse components and $\theta$ is the divergence of the beam with respect to a crystal alignment axis, then the angular aperture $\theta_{\text {coh }}$ for which the coherency condition holds can be estimated as

$$
\theta_{c o h} \lesssim \frac{\pi}{q_{\perp} d N} \sim \frac{1}{N}
$$

To an extent that the neutrino source can be treated as point-like, the angular divergence can be corrected for by proper alignment of elements of the multi-crystal converter.

\section{Acknowledgments:}

Thanks are due to N.N. Nikolaev for careful reading the manuscript and useful comments. Discussions with A.N. Rozanov and M.I. Vysotsky are greatfully acknowledged. 


\section{FIGURE CAPTIONS}

Fig.1

The upper bound on the non-diagonal neutrino magnetic moment in units of the Bohr

magneton as a function of the $W$-converter thickness is shown for different neutrino beam momenta. 


\section{References}

[1] V.R. Zoller, JETP Lett. 64 (1966) 788.

[2] I.Ya. Pomeranchuk and I.M. Shmushkevich, Nucl. Phys. 23 (1961) 452.

[3] A.Yu. Dubin, Sov.J.Nucl.Phys. 52 (1990) 790.

[4] V.V.Okorokov, Sov. J. Nucl. Phys. 2(1965) 719; JETP Lett. 2 (1965) 111. V.V. Okorokov, D.L Tolchenkov, Yu.P. Cheblukov et al., JETP Lett 16 (1972); Phys.Lett. A43 (1973) 485; V.V. Okorokov, JETP Lett., 62 (1995) 911.

[5] J. Schechter and J.W.F. Valle, Phys. Rev. D24 (1981) 1883;

P.B. Pal and L. Wolfenstein, Phys. Rev. D25 (1982) 766;

J. Nieves, Phys. Rev. D26 (1982) 3152;

M.B.Voloshin and M.I.Vysotsky, Sov. J. Nucl. Phys. 44(1986) 544;

M.B.Voloshin, M.I.Vysotsky and L.B. Okun', Sov. Phys. JETP 64 (1986) 446.

[6] M.C. Gonzalez-Garcia, F. Vannucci and J. Castromonte, Phys. Lett. B373 (1996) 153.

[7] S. Matsuki and K. Yamamoto, Phys. Lett. B289 (1992) 194.

[8] Makoto Sukuda, Phys. Rev. Lett. 72 (1994) 804.

[9] J.-M. Frere, R.B. Nevzorov and M.I. Vysotsky, Preprint ULB-TH/96/14.

[10] R.J. Glauber, in Lectures in Theoretical Physics, edited by W.E. Brittin et al., Interscience Publishers, Inc., New York, vol.1, p. 315, 1959.

[11] V.N. Gribov, Sov. Phys. JETP 29 (1969) 483; 30 (1970) 709.

[12] K. Gottfried, Ann. Phys. 66 (1971) 868

[13] D.S. Gemmel, Rev. Mod. Phys., 46 (1974) 1.

[14] D.O. Caldwell and R.N. Mohapatra, Phys. Rev. D48 (1993) 3259. 


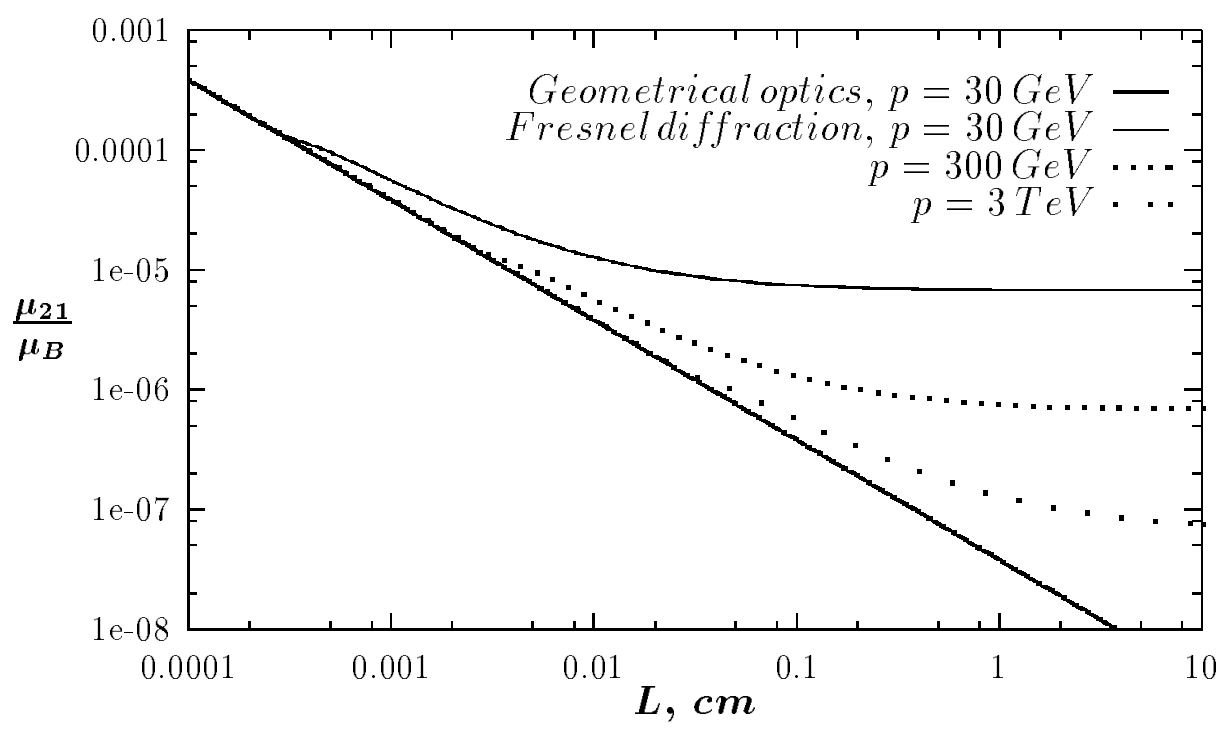

